\title{
Modeling of Spermbots in a Viscous Colloidal Suspension
}

\author{
Islam S. M. Khalil,* Anke Klingner, Veronika Magdanz, Friedrich Striggow, \\ Mariana Medina-Sánchez, Oliver G. Schmidt, and Sarthak Misra
}

Spermbots are biohybrid micromachines consisting of single sperm cells captured in artificial magnetic microstructures, and have the potential to act as autonomous tools for minimally invasive medicines and in diverse in vivo applications. This work investigates the hydrodynamic effects of the spermbots in a heterogeneous viscous medium similar to environments encountered in vivo. The propulsion of the spermbots is simulated using a numerical model based on the method of regularized Stokeslets for computing Stokes flows in the presence of immersed obstacles. It is shown that the concentration and size of these obstacles create a pressure gradient along the propulsion axis of the spermbot; hence they influence its effective net motion. In particular, the simulation results herein suggest that the forward and lateral swimming speeds of the spermbot increase with the concentration of the immersed obstacles and decrease with their size.

the fluid, while the latter provides a magnetic dipole moment for directional control under the influence of an external magnetic field. ${ }^{[6]}$ With sperm cells, even more so than with other microorganisms, we can prospectively navigate through fluids of the human body as they are not toxic. Spermbots were developed by capturing motile sperm cells in microtubes, which are fabricated by rolled up technology ${ }^{[7]}$ or 3D maskless lithography ${ }^{[0]}$ (Figure 1). Directional control under magnetic guidance, thermotriggered cell release, ${ }^{[10]}$ and remote-controlled transport and delivery of immotile sperm cells ${ }^{[1]]}$ as well as sperm-mediated drug delivery ${ }^{[5,12]}$ have been demonstrated using spermbots. In order to translate spermbots into in vivo

\section{Introduction}

There is a significant interest in the use of motile sperm cells and microorganisms to propel microobjects and achieve locomotion in viscous environments. The coupling between these cells or microorganisms and a magnetic structure enables us to develop biohybrid microrobots. ${ }^{[1-5]}$ The former imparts a propulsive force to

\section{Dr. I. S. M. Khalil, Prof. S. Misra}

Department of Biomechanical Engineering

University of Twente

7522 NB Enschede, The Netherlands

E-mail: i.s.m.khalil@utwente.nl

Dr. A. Klingner

The German University in Cairo

11835 New Cairo, Egypt

Dr. V. Magdanz, F. Striggow, Dr. M. Medina-Sánchez, Prof. O. G. Schmidt Institute for Integrative Nanosciences

Leibniz IFW

01069 Dresden, Germany

Dr. V. Magdanz

Applied Zoology

Technical University of Dresden

01062 Dresden, Germany

Prof. O. G. Schmidt

Center for Materials

Architectures and Integration of Nanomembranes, TU Chemnitz

09107 Chemnitz, Germany

Prof. S. Misra

Department of Biomedical Engineering

University of Groningen and University Medical Center Groningen

9713 AV Groningen, The Netherlands

The ORCID identification number(s) for the author(s) of this article can be found under https://doi.org/10.1002/adts.201900072

DOI: $10.1002 /$ adts.201900072 applications, the hydrodynamic effects in viscous media similar to that encountered in vivo should be studied. ${ }^{[13,14]}$ In the male and female reproductive tracts, sperm cells encounter fluids with a relatively large range of viscosities, up to several orders of magnitude greater than the viscosity of water. It has been described that sperm cells show a different motion pattern in highly viscous fluids which is derived from the difference in fluid mechanics. ${ }^{[15,16]}$ Berg and Turner have observed propulsion enhancement of microorganisms in solutions with highly structured viscous agents. ${ }^{[17]}$ They have suggested that the solution forms a loose-quasi-rigid network (gel-like polymer solution) and exerts normal forces to a body even when it does not possess a component of velocity along the normal direction. Leshansky has also presented a theoretical framework for propulsion through heterogeneous viscous media. ${ }^{[18]}$ This framework is based on solutions of averaged equations of viscous flow through a random spare array of obstructions (fibers or spheres). The numerical calculations have demonstrated that the propulsion is enhanced in terms of speed and efficiency owing to the random spare array of obstacles. Münch et al. have also modeled the flagellar swimming in complex environment by an array of obstacles and demonstrated that geometrical swimming leads to enhanced velocities. When these obstacles are not fixed, but being pushed aside, a reduced swimming speed is expected, as the moving obstacles display a reduced resistance to the pushing of the swimmer. ${ }^{[19]}$ Recently, modeling by multiparticle collision dynamics allowed a new explanation of the propulsion enhancement of bacteria on polymer solutions taking into account the polymer density distribution around the bacterial flagellum. ${ }^{[20]}$

Here, we develop a numerical model of the spermbot based on the method of regularized Stokeslets ${ }^{[21,22]}$ to determine the Stokes flows and the velocity of the spermbot in the presence 

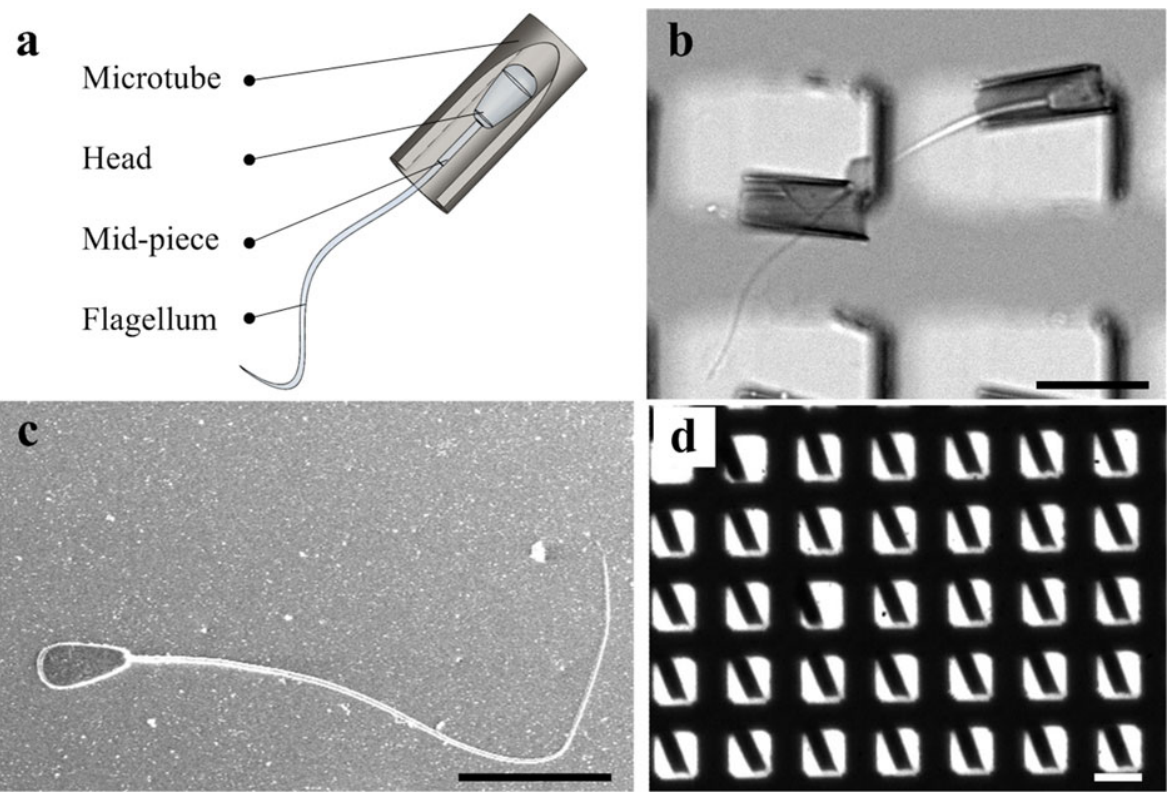

Figure 1. Spermbots consisting of rolled-up microtubes and bovine sperm cells. a) A schematic shows a sperm cell captured inside a microtube. b) Bright field microscopy images of bull sperm cells captured inside rolled up microtubes. c) A scanning electron microscopy image of a bull sperm. d) An array of rolled up $20-\mu \mathrm{m}$-long microtubes is fabricated by rolled up nanotechnology. ${ }^{[1,7]}$ Scale bars are $20 \mu \mathrm{m}$. Reproduced with permission. ${ }^{[8]}$ Copyright 2015, Wiley-VCH.

of immersed obstacles. This method enables us to compute Stokes flow driven by distributed forces at material points in a fluid based on the superposition of exact solutions of the Stokes equation. ${ }^{[23]}$ The physiological conditions encountered by the spermatozoa in vivo contain complex mixtures of suspended cells and viscous fluids. The female reproductive tract fluids contains secreted cells (e.g., epithelial cells, leucocytes, cell debris ${ }^{[24]}$ ) which are immersed in a highly viscoelastic matrix. In this work, we attempt to simulate this environment by taking into account the particle concentration and size and their effect on the resulting spermbot velocity and flagellar waveform. First, we use experimental flagellar beat patterns of the spermbots to determine the velocity of Stokeslets boundary points along the flagellum. Second, these velocities are used to calculate the flow-field of the spermbot and the obstacles. The theoretical prediction of this model shows the influence of the concentration and size of immersed obstacles in a colloidal suspension. In addition, the influence of the variation in the length of the mid-piece and the depth of the sperm head inside the tube on the propulsion are investigated.

\section{Governing Equations of the Spermbot in a Viscous Colloidal Suspension}

We consider a tube with an average magnetic moment $\mathbf{M}$, lying along its long axis, of length $L_{\mathrm{t}}$ and radius $R_{\mathrm{t}}$. This magnetic moment enables directional control under the influence of an external magnetic field B. A sperm cell with spheroidal head is rigidly attached to the inner surface of the tube at a distance $d$, as shown in Figure 2a. The spheroidal head of the sperm cell has major diameter $2 R_{\text {maj }}$ and minor diameter $2 R_{\min }$. This head is rigidly attached to a flexible flagellum via a rigid mid-piece with length $L_{\mathrm{m}}$. The flagellum of length $L_{\mathrm{f}}$ and diameter $2 R_{\mathrm{f}}$, oscillates at an angular frequency $\omega$. The resulting spermbot is contained in a viscous colloidal suspension with viscosity $\mu$ and density $\rho$ (Figure $2 \mathrm{~b}$ ). The colloidal suspension contains particles with average diameter $2 R_{\mathrm{p}}$ and area fraction $\phi=\frac{\pi R_{\mathrm{p}}^{2} N_{\mathrm{p}}}{A}$, where $N_{\mathrm{p}}$ is the number of particles and $A$ is their total area. The spermbot undergoes flagellar swimming at an average forward speed $U_{x}$, while immersed in the colloidal suspension, characterized by low Reynolds number ( $R e=\frac{\rho U_{x} L}{\mu}$ ) hydrodynamics on the order of $\mathcal{O}\left(10^{-3}\right)$, where $L$ is the characteristic length of the spermbot. The governing fluid mechanics equations for the spermbot at low Reynolds numbers are given by the following Stokes equation:

$$
\begin{aligned}
& \mu \nabla^{2} \mathbf{u}+\mathbf{f}-\nabla p=0 \\
& \nabla \cdot \mathbf{u}=0
\end{aligned}
$$

where $\mathbf{u}$ is the velocity vector field. Further, $\mathbf{f}$ and $p$ are the body force of the spermbot acting on the fluid and the scalar pressure field, respectively. The pressure field is influenced by the size and concentration of the immersed obstacles of the medium. The surface of the microtube, the head and flagellum of the sperm, and the particles of the colloidal suspension are covered with $N$ Stokeslets boundary points. The position and velocity of the $i$ th boundary point is $\mathbf{r}_{i}=\left(x_{i}, y_{i}\right)$ and $\mathbf{u}_{i}$, respectively, for $i=1, \ldots, N$ The distance between the boundary points is $\Delta s$. To investigate the local flow-field of the spermbot, a numerical simulation based on the method of regularized Stokeslets is used. This 


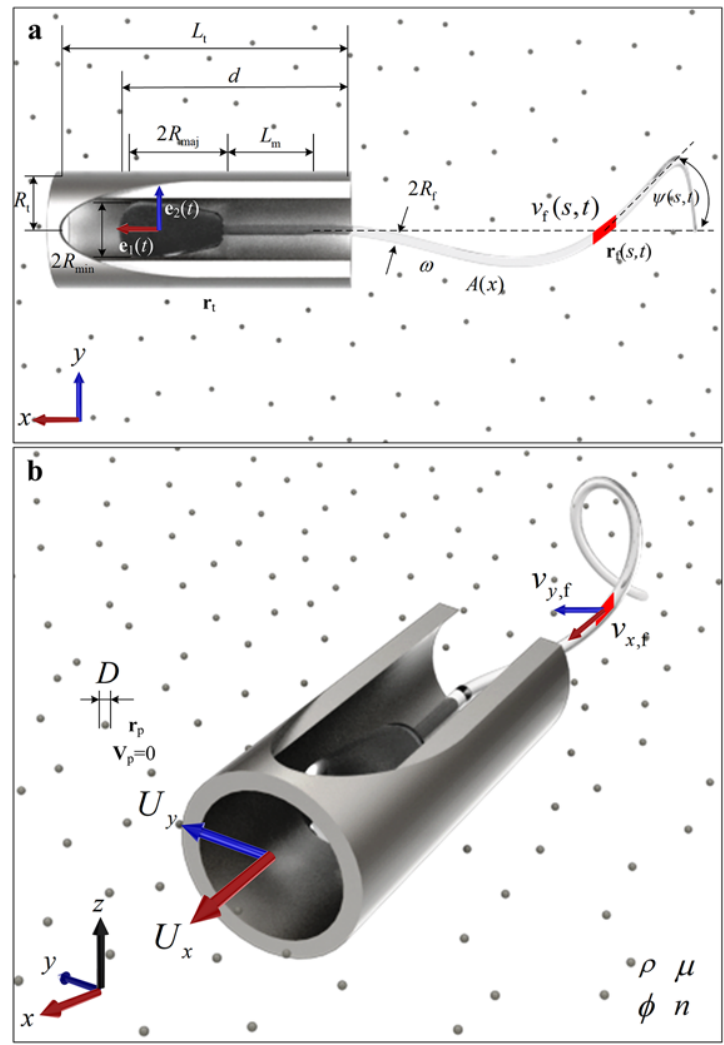

Figure 2. A schematic representation shows a spermbot in a viscous colloidal suspension. a) The spheroidal head of the sperm cell is fixed to the inner surface of the microtube at distance $d$. b) Motion of the spermbot is governed by the local equilibrium between the viscous and elastic forces. The particles in the colloidal suspension are modeled as obstructions, each exert mean drag force of $6 \pi \mu r R_{\mathrm{p}} \mathbf{u}$, where $\mathbf{u}$ is the uniform flow.

method is based on the superposition of the following delta functions:

$\mathbf{F}(\mathbf{r})=\mathbf{f}_{0} \phi_{\epsilon}\left(\mathbf{r}-\mathbf{r}_{0}\right)$

where $\phi_{\epsilon}(\mathbf{r})$ is a cutoff function centered at the point $\mathbf{r}_{0}$ and given by

$\phi_{\epsilon}(\mathbf{r})=\frac{3 \epsilon^{3}}{2 \pi\left(|\mathbf{r}|^{2}+\epsilon^{2}\right)^{5 / 2}}$

In (4), $\epsilon$ is a parameter that describes the sharpness of the delta function, and is dependent on the distance between the boundary points using, $\epsilon=0.25 \Delta$ s. Based on the regularized Stokeslet method, the pressure, due to force $\mathbf{f}_{k}$ at points $\mathbf{x}_{k}$, is determined as follows:

$p(\mathbf{x})=\sum_{k=1}^{N} \frac{1}{2 \pi}\left[\mathbf{f}_{k} \cdot\left(\mathbf{x}-\mathbf{x}_{k}\right)\right]\left[\frac{r_{k}^{2}+2 \epsilon^{2}+\epsilon \sqrt{r_{k}^{2}+\epsilon^{2}}}{\left(\sqrt{r_{k}^{2}+\epsilon^{2}}+\epsilon\right)\left(r_{k}^{2}+\epsilon^{2}\right)^{3 / 2}}\right]$ where $r_{k}=\left|\mathbf{x}-\mathbf{x}_{k}\right|$. Further, the velocity due to force $\mathbf{f}_{k}$ at points $\mathbf{x}_{k}$ is given by

$$
\begin{aligned}
& \mathbf{u}(\mathbf{x})= \\
& \sum_{k=1}^{N} \frac{-\mathbf{f}_{k}}{2 \pi \mu}\left[\ln \left(\sqrt{r_{k}^{2}+\epsilon^{2}}+\epsilon\right)-\frac{\epsilon\left(\sqrt{r_{k}^{2}+\epsilon^{2}}+2 \epsilon\right)}{\left(\sqrt{r_{k}^{2}+\epsilon^{2}}+\epsilon\right) \sqrt{r_{k}^{2}+\epsilon^{2}}}\right]_{(6)} \\
& +\frac{1}{4 \pi \mu}\left[\mathbf{f}_{k} \cdot\left(\mathbf{x}-\mathbf{x}_{k}\right)\right]\left(\mathbf{x}-\mathbf{x}_{k}\right)\left[\frac{\sqrt{r_{k}^{2}+\epsilon^{2}}+2 \epsilon}{\left(\sqrt{r_{k}^{2}+\epsilon^{2}}+\epsilon\right)^{2} \sqrt{r_{k}^{2}+\epsilon^{2}}}\right]
\end{aligned}
$$

Equation (6) can be used directly to compute the flow due to given forces. It is also possible to compute the forces that create the flow-field when the velocity of the beating flagellum is known. Therefore, Equation (6) can be written as a system of equations as follows:

$\mathbf{u}\left(\mathbf{x}_{i}\right)=\sum_{j=1}^{N} M_{i j}\left(\mathbf{x}_{1}, \ldots, \mathbf{x}_{N}\right) \mathbf{f}_{j}$, for $i=1, \ldots, N$

Equation (7) can also be represented in the following compact form:

$\mathcal{U}=\mathcal{M F}$

where $\mathcal{U}$ is a $2 N \times 1$ velocity vector and $\mathcal{F}$ is a $2 N \times 1$ force vector. Further, $\mathcal{M}$ is a $2 N \times 2 N$ matrix. We define Stokeslet points on the beating flagellum of the spermbot as a function of the arc length $s$ as follows: ${ }^{[25]}$

$x_{\mathrm{f}}(s, t)=-\left(r_{\mathrm{h}}+L_{\mathrm{m}}\right) \mathbf{e}_{1 x}(t)-\sum_{0}^{s} \cos \psi(s, t) \Delta s \pm \sin \psi(s, t) R_{\mathrm{f}}$

$\gamma_{\mathrm{f}}(s, t)=-\left(r_{\mathrm{h}}+L_{\mathrm{m}}\right) \mathbf{e}_{1 y}(t)-\sum_{0}^{s} \sin \psi(s, t) \Delta s \pm \cos \psi(s, t) R_{\mathrm{f}}$

where $\psi(s, t)$ is the tangent angle at each flagellum point enclosed by the long axis of the sperm head and the local tangent of the flagellum at position $\mathbf{r}(s, t)$. Further, $\mathbf{e}_{1 x}(t)$ and $\mathbf{e}_{1 \gamma}(t)$ are timedependent unit vectors that are initially oriented along the major $\left(\mathbf{e}_{1}\right)$ and minor $\left(\mathbf{e}_{2}\right)$ axes of the head, respectively, and change each time step $\Delta t$ as follows:

$\dot{\mathbf{e}}_{1}(t)=\Omega(t) \Delta t \mathbf{e}_{2}(t)$

$\dot{\mathbf{e}}_{2}(t)=-\Omega(t) \Delta t \mathbf{e}_{1}(t)$

where $\Omega(t)=\hat{\Omega} \cos \omega t$ is the angular velocity of the sperm head with amplitude $\hat{\Omega}$. In (9), $\psi(s, t)$ is the curvature of the flagellum and is governed by

$\psi\left(s+L_{m}\right)=\psi_{0}(s)$

$+2 \psi_{1}(s) \cos [\phi(s)] \cos (\omega t)+2 \psi_{1}(s) \sin [\phi(s)] \sin (\omega t)$ 

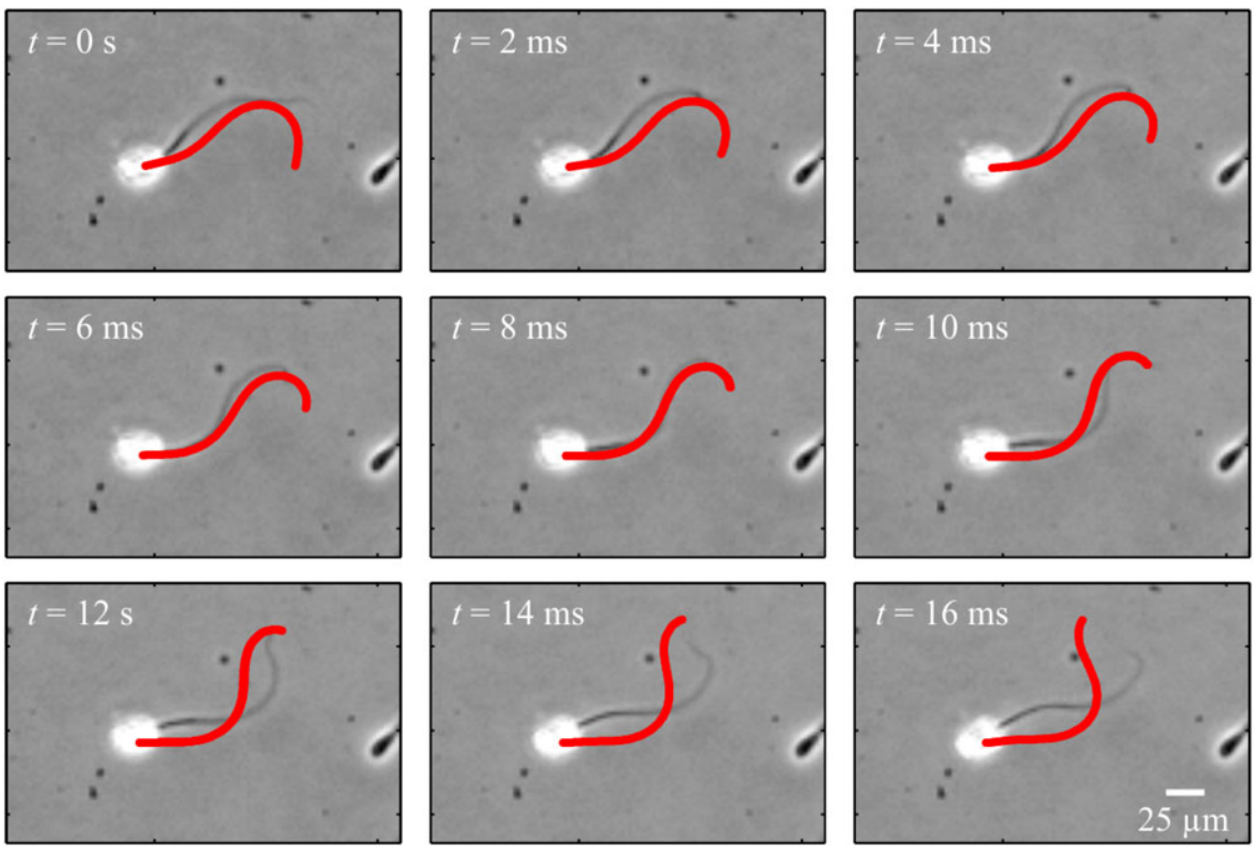

Figure 3. Calculated versus measured deformations of the flagellum of the spermbot are provided at different time instances. The tail deformation (red line) is calculated using Equation (11) for $K_{0}=1500 \mathrm{~m}^{-1}$ (mean flagellar curvature), $A_{0}=-14000 \mathrm{~m}^{-1}$ (amplitude), $\lambda=150 \mu \mathrm{m}$ (wavelength), $T=$ $0.138 \mathrm{~s}, \hat{\Omega}=15 \mathrm{l} / \mathrm{s}$ (angular frequency), $R_{\text {maj }}=R_{\min }=3 \mu \mathrm{m}$ (head diameter), $L_{\mathrm{f}}=100 \mu \mathrm{m}$ (flagellum length), and $R_{\mathrm{f}}=0.25 \mu \mathrm{m}$ (flagellum radius). Motion of the spermbot is observed using phase-contrast microscopy at a rate of $500 \mathrm{fps}$, at $37^{\circ} \mathrm{C}$ in SP-TALP (modified tyrode's albumin lactate pyruvate medium)

In (11), $\psi_{0}(s)=K_{0} s$, where $K_{0}$ is the mean flagellar curvature $\left(K_{0}=13.1 \times 10^{3} \mathrm{~m}^{-1}\right)$. Further, $\psi_{1}(s)=A_{0} s$ and the amplitude of the flagellar beat is $A_{0}=14.6 \times 10^{3} \mathrm{~m}^{-1}$. In $(11), \phi(s)=\frac{2 \pi}{\lambda} s$, where $\lambda$ is the wavelength of the flagellar bending wave $(\lambda=$ $66.6 \times 10^{-6} \mathrm{~m}$ ). The amplitude of the flagellar beat and angular frequency are reduced if there is overlap between the tube and the flagellum. The velocities of the boundary points on the flagellum are approximated using:

$v_{x, f}=\frac{x_{f}(s, t+\Delta t)-x_{f}(s, t)}{\Delta t}$ and

$v_{\gamma, f}=\frac{\gamma_{f}(s, t+\Delta t)-\gamma_{f}(s, t)}{\Delta t}$

Each particle in the colloidal suspension is also represented by Stokeslets boundary points along its circumference. These Stokeslets points are separated by distance $6 \Delta s$. The diameter of the particles is controlled by the location of the Stokeslets boundary points along its circumference. The initial positions of the particles are randomly arranged within the vicinity of the spermbot. The position of these particles is $\left(x_{\mathrm{p} i}, \gamma_{\mathrm{p} i}\right)$, for $i=$ $1, \ldots, N_{\mathrm{p}}$, with zero initial velocity $\left(u_{x}=u_{\gamma}=0\right)$. The flagellum movement $\left(v_{x}, v_{y}\right)$ induces motion of the whole spermbot $\left(U_{x}\right.$, $\left.U_{Y}\right)$. For the Stokeslet points on the flagellum, $u_{x}=v_{x}+U_{x}$ and $u_{Y}=v_{y}+U_{y}$. Forward speed $U_{x}$ and lateral speed $U_{Y}$ are varied until the sum of forces in Equation (8) on the spermbot (head, flagellum, and microtube) is zero.
Table 1. Characteristics of the spermbot and the colloidal suspension.

\begin{tabular}{lccccc}
\hline Parameter & Value & Parameter & Value & Parameter & Value \\
\hline$R_{\text {min }}[\mu \mathrm{m}]$ & 2.5 & $R_{\text {maj }}[\mu \mathrm{m}]$ & 4.5 & $R_{\mathrm{f}}[\mu \mathrm{m}]$ & 0.25 \\
$L_{\mathrm{m}}[\mu \mathrm{m}]$ & $0-30$ & $d[\mu \mathrm{m}]$ & $0-25$ & $L_{\mathrm{t}}[\mu \mathrm{m}]$ & 25 \\
$R_{\mathrm{t}}[\mu \mathrm{m}]$ & 5 & $L_{\mathrm{f}}[\mu \mathrm{m}]$ & 60 & $D[\mu \mathrm{m}]$ & $2-10$ \\
$\mu[\mathrm{mPa} s]$ & 0.7 & $\rho\left[\mathrm{kg} \mathrm{m}^{-3}\right]$ & 1000 & $\phi[\%]$ & $0.5-2$ \\
\hline
\end{tabular}

\section{Simulation Results}

The flagellated propulsion of the spermbot is simulated to analyze the influence of the concentration of the particles in the colloidal suspension and the size of these particles (Table 1). In addition, geometric aberrations between spermbots are experimentally observed owing to spermbot-to-spermbot variability in the depth of the head in the microtube and the length of the midpiece of the sperm cells. Therefore, we study the geometric aberrations of the spermbots and external pressure of the immersed obstacles.

Spermbots are obtained and allowed to swim in SP-TALP (Sperm Tyrode's Albumin Lactate Pyruvate) medium of viscosity $\mu=0.7 \mathrm{mPa}$ s at $37^{\circ} \mathrm{C}$ inside a $25 \times 15 \times 0.15 \mathrm{~mm}^{3}$ chamber. These spermbots are constituted by bull sperm cells and synthetic cylindrical caps. Swimming of the spermbots is observed using phase-contrast microscopy (Zeiss Axio Observer.A1) with a $63 \times$ objective. The deformation of the flagellum is recorded at a rate of $500 \mathrm{fps}$ using a high speed camera (Vision Research Phantom Miro eX2), as shown in Figure 3. Equation (9) is used to 

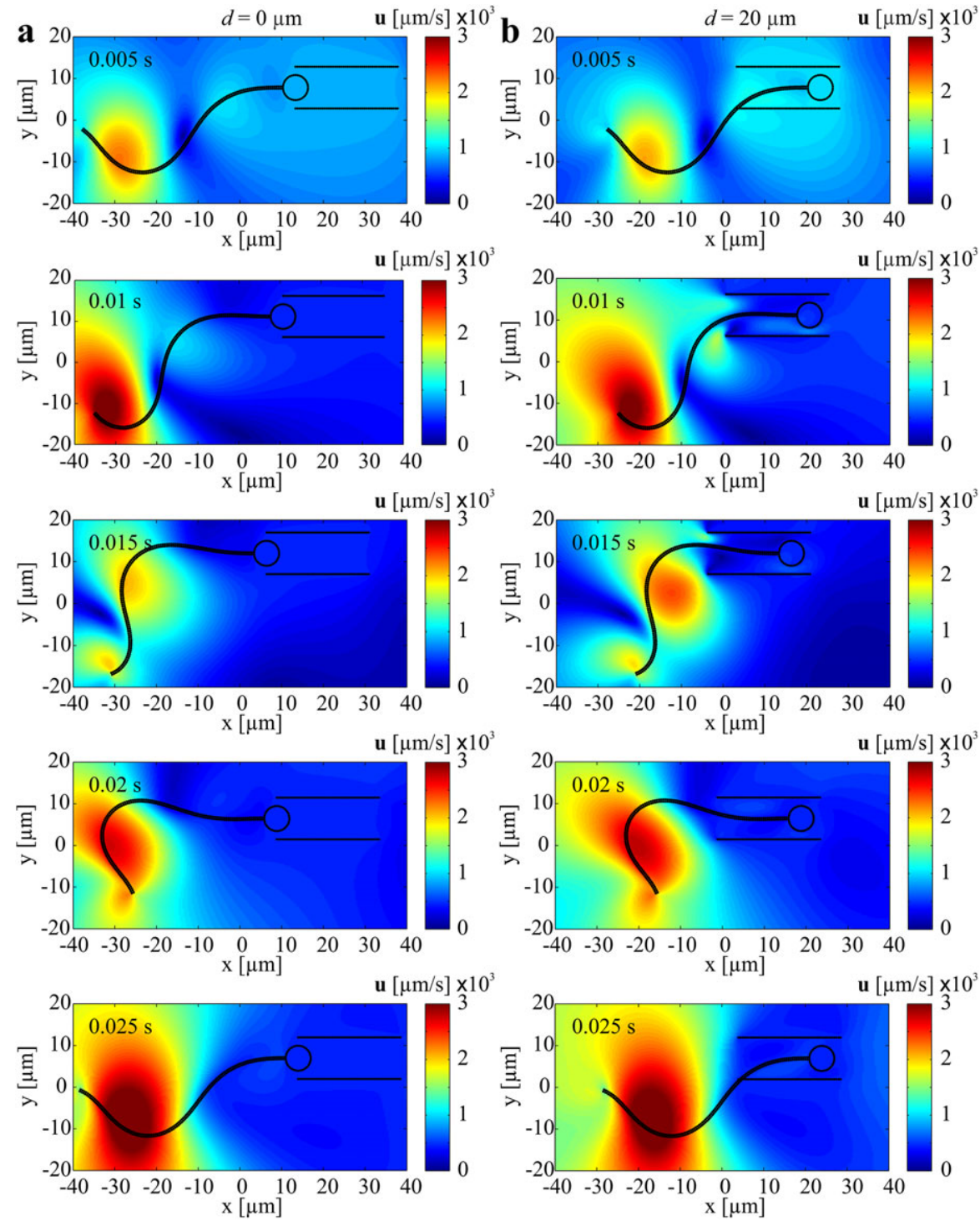

Figure 4. Influence of the depth of the sperm head inside the microtube for depth of 0 and $20 \mu \mathrm{m}$. The average speed of the spermbot decreases with the depth of the sperm head inside the microtube. Parameters: $L_{m}=0 \mu \mathrm{m}$ and $\varphi=0 \%$. a) At depth $d=0 \mu \mathrm{m}$, the forward and lateral speeds are 119 and $44 \mu \mathrm{m} \mathrm{s}^{-1}$, respectively. b) At depth $d=20 \mu \mathrm{m}$, the forward and lateral speeds are 83 and $65 \mu \mathrm{m} \mathrm{s}^{-1}$, respectively.

predict the deformation of the tail of the spermbot for $K_{0}=1500$ $\mathrm{m}^{-1}, A_{0}=-14000 \mathrm{~m}^{-1}, \lambda=150 \mu \mathrm{m}, T=0.138 \mathrm{~s}, \hat{\Omega}=151 / \mathrm{s}$, $R_{\text {maj }}=R_{\text {min }}=3 \mu \mathrm{m}, L_{\mathrm{f}}=100 \mu \mathrm{m}$, and $R_{\mathrm{f}}=0.25 \mu \mathrm{m}$. Qualitative agreement between the predicted deformation of the flagellum and the measurements is observed for one beat cycle of the spermbot. Therefore, Equation (11) provides a valid approximation of the deformation of the flagellum inside the microtube and is used to determine the velocities of the Stokeslets boundary point along the flagellum.

\subsection{Influence of the Depth of the Sperm Head inside the Microtube and Mid-Piece}

Spermbots consist of a microtube that captures a single motile sperm cell, as shown in Figure 1. The depth of the sperm head inside the microtube varies based on the length and diameter of the microtube ${ }^{1}$. This depth plays a significant role in determining the swimming speed due to the restriction of the amplitude of the flagellar bending wave by the microtube walls.

Therefore, we vary the depth $(d)$ of the sperm head inside the microtube between 0 to $20 \mu \mathrm{m}$. Figure $4 \mathrm{a}$, b shows the behavior of the spermbot over one beat cycle for a depth of 0 and $20 \mu \mathrm{m}$, respectively. In the case of $d=0 \mu \mathrm{m}$, the complete flagellum acts on the fluid and provides a maximum flow-field at the distal tip of the flagellum. As the penetration depth of the sperm head increases inside the microtube, the flow-field due to the distal tip of the flagellum decreases, and an asymmetric flow-field is created along the lateral direction of the microtube. As a consequence of these two local flow-fields, the lateral speed of the spermbot increases with the depth of the sperm head inside the microtube. This behavior is demonstrated in Figure 5. The forward and 


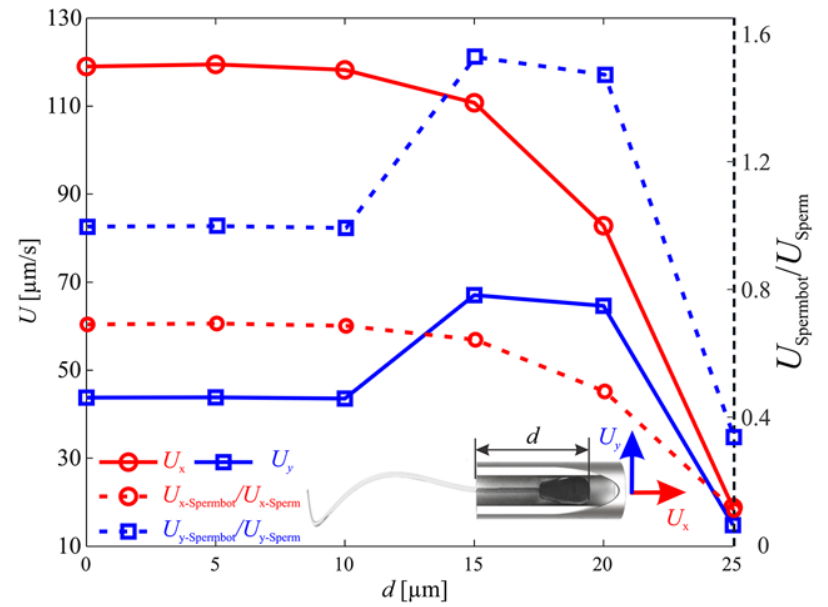

Figure 5. The forward $\left(U_{x}\right)$ and lateral $\left(U_{\gamma}\right)$ speeds of the spermbot are calculated versus the depth of the sperm head $(d)$ inside the microtube. The forward speed decreases with the depth of the sperm head inside the microtube, while the lateral speed increases for $10<d<20 \mu \mathrm{m}$. Normalized forward and lateral speeds are plotted in dashed lines (right $y$ axis, normalized to velocities of free sperm). Parameters: $L_{m}=0 \mu \mathrm{m}$ and $\varphi=0 \%$.

lateral speeds of the spermbot and sperm cell are calculated for different penetration depths in the absence of any immersed obstacles in the medium. In the case of $d=0 \mu \mathrm{m}$, the average forward and lateral speeds (over one beat cycle) of the spermbot are approximately 119 and $44 \mu \mathrm{m} \mathrm{s}^{-1}$, respectively. The forward speed of the spermbot decreases to $110 \mu \mathrm{m} \mathrm{s}^{-1}$ for $d=15 \mu \mathrm{m}$, while its lateral speed increases to $67 \mu \mathrm{m} \mathrm{s}^{-1}$. As the penetration depth of the sperm head increases inside the microtube from 15 to $20 \mu \mathrm{m}$, the forward and lateral speeds decrease to approximately 83 and $65 \mu \mathrm{m} \mathrm{s}^{-1}$, respectively. At $d=25 \mu \mathrm{m}$, the forward and lateral speeds decrease to 18 and $15 \mu \mathrm{m} \mathrm{s}^{-1}$, respectively. The normalized lateral speed curve $U_{\mathrm{y} \text {-spermbot }} / U_{\mathrm{y} \text {-sperm }}$ (Figure 5 ) shows the improved speed range between 15 and $20 \mu \mathrm{m}$ of penetration depth, where the spermbot's lateral speed exceeds the free sperm speeds, indicated by a normalized speed above one. In contrast to lateral speed, the normalized forward speed ( $\left.U_{\mathrm{x} \text {-spermbot }} / U_{\mathrm{x} \text {-sperm }}\right)$ decreases regardless to the depth of the head inside the tube.

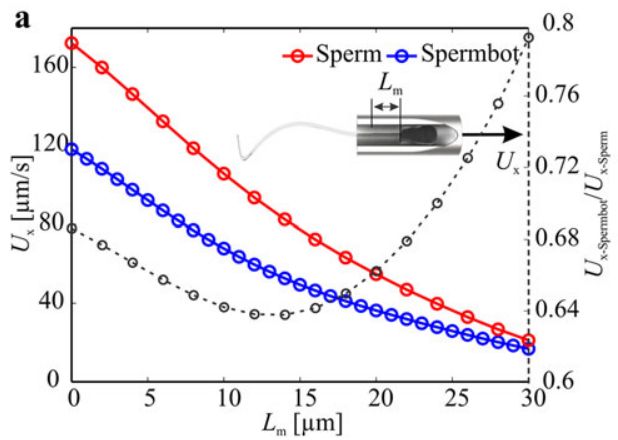

Figure 6. The forward $\left(U_{x}\right)$ and lateral $\left(U_{\gamma}\right)$ speeds of the sperm cell and spermbot are calculated versus the length of the mid-piece $\left(L_{\mathrm{m}}\right)$ of the sperm cell. a) The forward speed of the sperm and spermbots decreases with the length of the mid-piece. b) The lateral speed of the sperm and the spermbots also decrease with $L_{m}$. Normalized speeds are plotted over the right $\gamma$-axes (normalized to average free sperm speed for each mid-piece length). Parameters: $d=10 \mu \mathrm{m}$ and $\varphi=0 \%$.

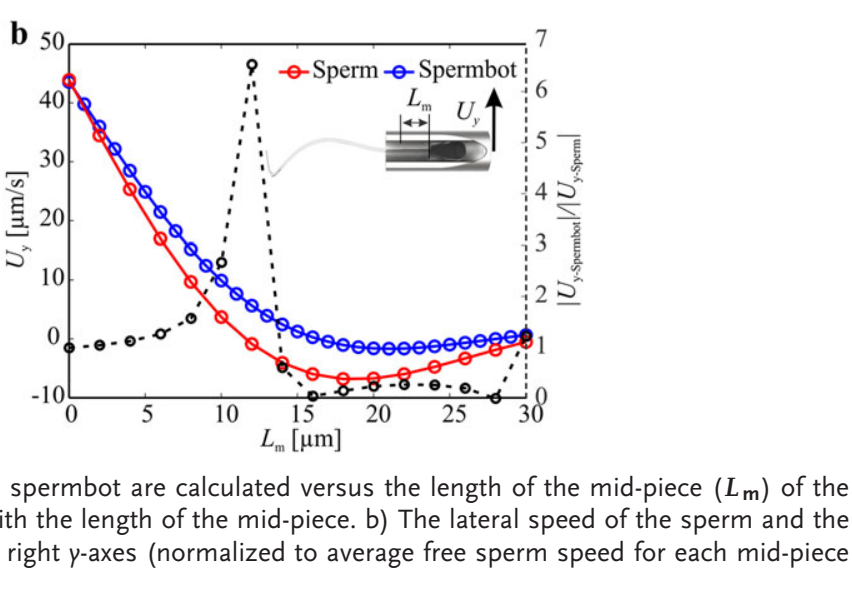

In another series of simulations, we vary the length of the mid-piece of the sperm cell and determine the forward and lateral speeds of the sperm cell and the spermbot, as shown in Figure $\mathbf{6 a}, \mathrm{b}$, respectively. It is unlikely that the length of the mid-piece changes much between sperm cells of the same species. ${ }^{[26]}$ However, this length varies between species that can be used to provide propulsive force to the spermbots. As expected the forward speed of the spermbot is smaller than that of the free sperm cell regardless of the length of the mid-piece $\left(0 \leq L_{\mathrm{m}} \leq 30 \mu \mathrm{m}\right)$. The forward speed of the spermbot decreases from $86 \%$ to $14 \%$ (Figure 6a), whereas the lateral speed decreases by $99 \%$ (Figure $6 \mathrm{~b}$ ). The normalized forward speed of the spermbot to the sperm $\left(U_{\mathrm{x} \text {-spermbot }} / U_{\mathrm{x} \text {-sperm }}\right)$ indicates that the sperm cell has greater forward speed than the spermbot he spermbot to sperm $\left(U_{\mathrm{y} \text {-spermbot }} / U_{\mathrm{y} \text {-sperm }}\right)$ shows th their lateral speeds are approximately equal. At $L_{\mathrm{m}}=12 \mu \mathrm{m}$, the ateral speed of the sperm is close to zero and thereby resulting the peak shown in Figure $6 \mathrm{~b}$

The flow-field of the spermbot is a combination of the Stokeslets fields of the flagellar thrust and the associated to the drag caused by the sperm head, the mid-piece, and the microtube. Therefore, the forward speed of the spermbot decreases owing to the increased drag by the mid-piece for the same flagellar thrust.

\subsection{Influence of the Concentration of the Immersed Obstacles}

For spermbots swimming within a colloidal suspension, the pressure and velocity fields are influenced by the concentration and size of the obstacles. It has been shown that the stationary spherical obstructions with diameter $D$ result in a pressure field $\nabla p=3 n \pi \mu D \mathrm{U}$, where $n$ is the number density of obstructions within the colloidal suspension. ${ }^{[18]}$ We allow the immersed particles to have a uniformly distributed random positions within the suspension for each concentration and particle size. Figure $7 \mathrm{a}-\mathrm{c}$ show the behavior of the spermbot over one beat cycle for $\varphi=0,1,2 \%$, respectively. In the absence of any immersed obstacles, the flow-field is generated by the Stokeslets field of the flagellar thrust only (Figure 7a). For a non-zero $\varphi$, the flow-field is a combination of the Stokeslets fields of the flagellar thrust and the local fields associated with the drag caused by the immersed obstacles (Figure 7b,c). We allow the concentration to 

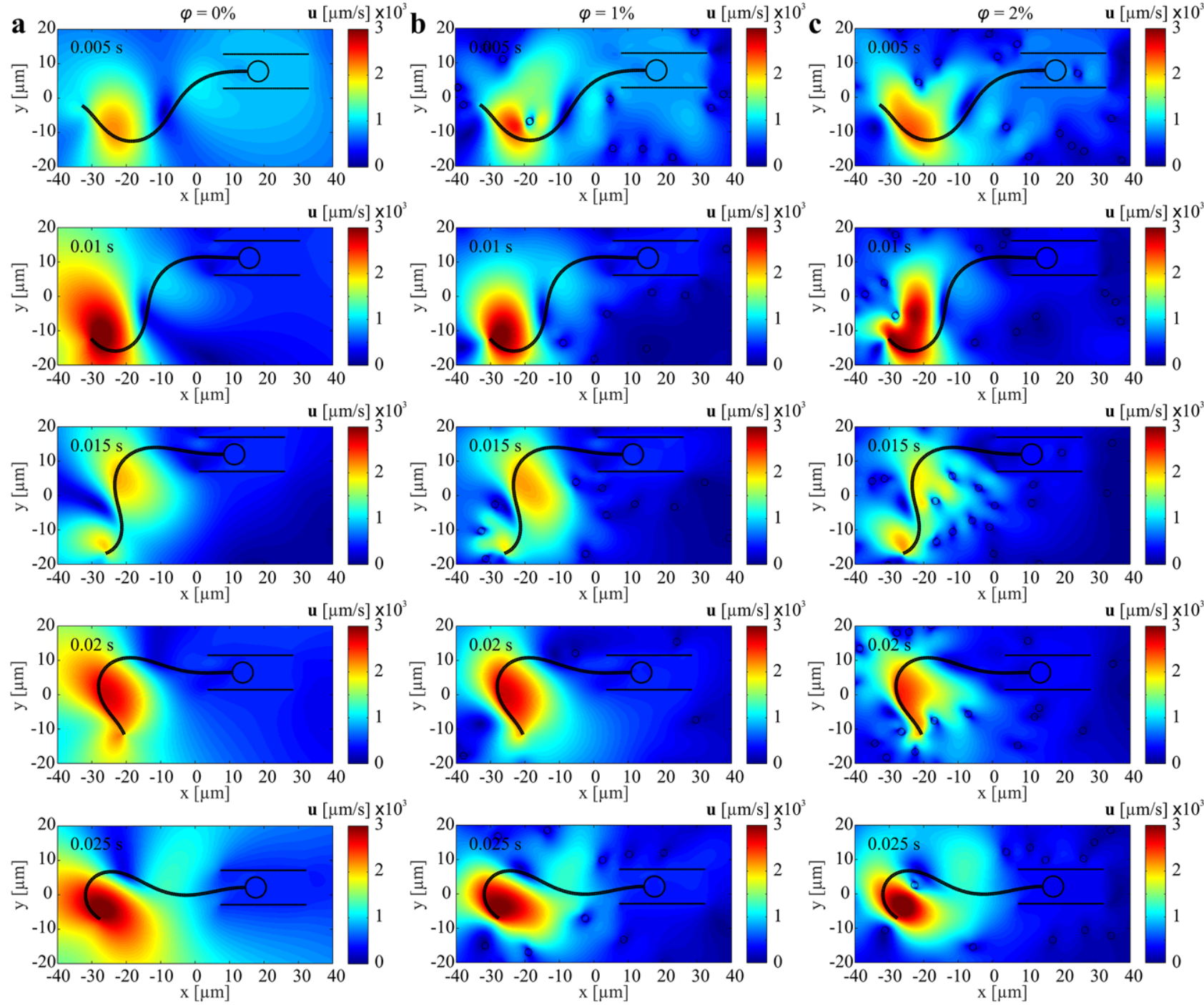

Figure 7. Influence of the concentration of the particles in the colloidal suspension on the flagellar propulsion of the spermbot is calculated for concentrations of 0,1 , and 2 . The average speed of the spermbot increases with the concentration of the particles in the colloidal suspension. Parameters: $D=1 \mu \mathrm{m}, L_{\mathrm{m}}=0 \mu \mathrm{m}$, and $d=10 \mu \mathrm{m}$. a) At $\varphi=0, U_{x}=31.2$ and $U_{y}=85.8 \mu \mathrm{m} \mathrm{s}^{-1}$. b) At $\varphi=1, U_{x}=68.1$, and $U_{y}=81.4 \mu \mathrm{m} \mathrm{s}{ }^{-1}$. c) At $\varphi=2$, $U_{x}=127.2$ and $U_{y}=79.6 \mu \mathrm{m} \mathrm{s}^{-1}$.

vary $(0 \leq \varphi \leq 0.02)$ for spherical particles with diameter of $1 \mu \mathrm{m}$ and calculate the forward and lateral speeds of the free sperm cell and spermbot, as shown in Figure 8. We observe that the forward and lateral speeds of the sperm cell and spermbot increase with the concentration of the immersed spherical particles. The forward swimming speeds of the free sperm cell increase from 172 to $364 \pm 54 \mu \mathrm{m} \mathrm{s}^{-1}(n=10)$ when the concentration is increased by $1 \%$, whereas the speed of the spermbot increases from 118 to $268 \pm 42 \mu \mathrm{m} \mathrm{s}^{-1}(n=10)$, as shown in Figure 8a. When the particle concentration is increased from $0 \%$ to $1 \%$, the lateral speed of the sperm and spermbot changes from 44 to $81 \pm 30 \mu \mathrm{m} \mathrm{s}^{-1}(n=10)$ and from 43 to $37 \pm 29 \mu \mathrm{m} \mathrm{s}^{-1}(n=10)$, respectively (Figure $\left.8 \mathrm{~b}\right)$. For $\varphi=2 \%$, the lateral speeds of the sperm and spermbot increase to $77 \pm 6$ and $59 \pm 21 \mu \mathrm{m} \mathrm{s}^{-1}$, respectively. Our simulation results show propulsion enhancement for the sperm and spermbot with the concentration of the obstacles. These obstacles are uniformly distributed within the medium and influence the microtube and the beating flagellum. However, the velocity of the flow-field in close proximity to the flagellum is greater than the flow-field near the head with approximately 3 orders of magnitude, as show in Figure 7. Therefore, the flagellum is influenced by greater pressure than the microtube since the pressure gradient depends on the concentration and velocity of the flow-field.

\subsection{Influence of the Size of the Immersed Obstacles}

The pressure field is influenced by the concentration of the particles and their size using, $\nabla p=3 n \pi \mu D \mathrm{U}$. Therefore, the diameter of the particles is varied between 1 and $10 \mu \mathrm{m}$, and 

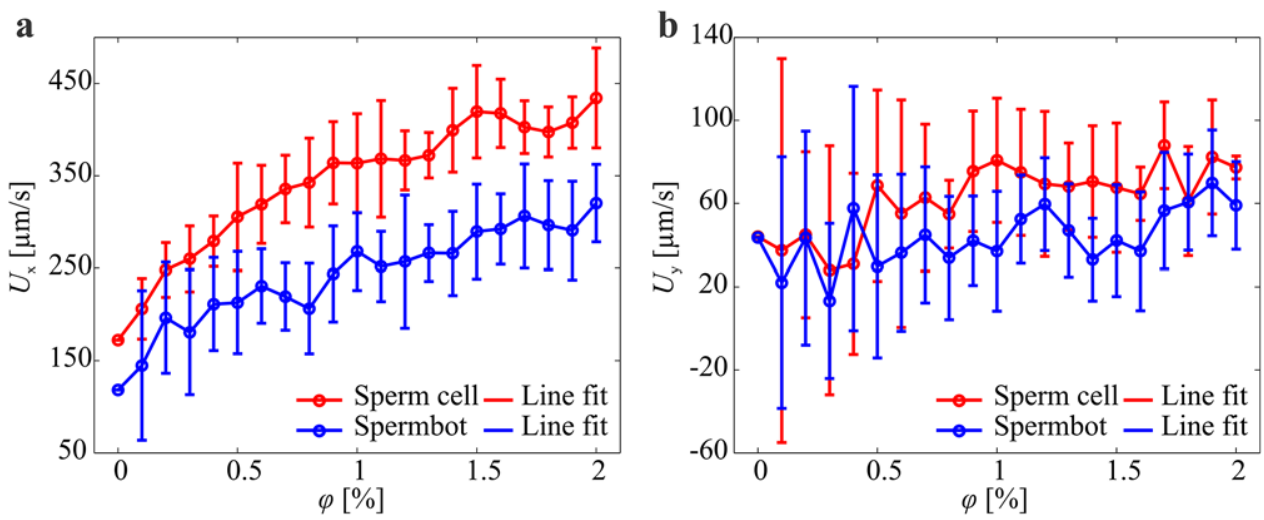

Figure 8. Forward and lateral speeds $\left(U_{x}\right.$ and $\left.U_{y}\right)$ of the sperm and spermbots are calculated versus the concentration $(\varphi)$ of the particles in the colloidal suspension. The particles are arranged randomly and the average speed and standard deviation are calculated for ten simulations. Parameters: $D=1 \mu \mathrm{m}, L_{\mathrm{m}}=0 \mu \mathrm{m}$, and $d=10 \mu \mathrm{m}$. a) The average forward speed of the sperm cell and spermbot increases by $47 \%$ and $51 \%$ for $\varphi=1 \%$, respectively. b) The average lateral speed of the sperm cell and spermbot increases by $50 \%$ and $43 \%$ for $\varphi=1 \%$, respectively.

the corresponding flow-fields are simulated for one complete beat cycle (Figure 9). We allow the initial position of the immersed particles to vary randomly in each simulation. Figure 9a,b show the flow-fields of the spermbot for immersed spherical particles with concentration of $1 \%$ and diameter of 1 and $5 \mu \mathrm{m}$, respectively.

In contrast to the concentration of the immersed obstacles, their size does not enhance the flagellar propulsion of the sperm cell or the spermbot. Figure 10a shows the forward speed of the sperm cell and spermbot for $2<D<10 \mu \mathrm{m}$. We observe a linear decrease of the forward speed of the sperm and spermbot with the increasing diameter, for a fixed concentration of $1 \%$. In the case of $D=2 \mu \mathrm{m}$, the average forward speed of the sperm and spermbots are calculated as $364 \pm 53$ and $260 \pm 57 \mu \mathrm{m}$ $\mathrm{s}^{-1}$, respectively. Their forward speeds decrease to $183 \pm 46$ and $128 \pm 50 \mu \mathrm{m} \mathrm{s}^{-1}$, for $D=10 \mu \mathrm{m}$. Figure $10 \mathrm{a}$ also shows the effect of increasing the size of the spherical obstacles on the ratio between the normal $\left(C_{n}\right)$ and tangential $\left(C_{1}\right)$ drag coefficients of the flagellum. These coefficients are given by ${ }^{[18]}$

$$
\begin{aligned}
C_{\mathrm{n}} & =4 \pi \mu\left(\frac{1}{4}\left(\alpha R_{\mathrm{p}}\right)^{2}+\alpha R_{\mathrm{p}} \frac{K_{1}\left(\alpha R_{\mathrm{p}}\right)}{K_{0}\left(\alpha R_{\mathrm{p}}\right)}\right) \text { and } \\
C_{1} & =4 \pi \mu\left(\frac{1}{2} \alpha R_{\mathrm{p}} \frac{K_{1}\left(\alpha R_{\mathrm{p}}\right)}{K_{0}\left(\alpha R_{\mathrm{p}}\right)}\right)
\end{aligned}
$$

where $K_{i}\left(\alpha R_{\mathrm{p}}\right)$ is the modified Bessel function of degree $i$. The ratio between these drag coefficients must be greater than 1 to achieve forward propulsion. ${ }^{[17]}$ Therefore, the size of the immersed obstacles does not increase this ratio for $>2 \mu \mathrm{m}$, as shown in Figure 10a. Unlike the forward speed of the spermbot, the lateral speed is not affected by the size of the immersed obstacles (Figure 10b). However, we observe a fundamental difference between the influence of the concentration and size of immersed obstacles on the forward and lateral speeds. The uncertainties in the calculated lateral speed (Figures $8 \mathrm{~b}$ and 10b) are relatively greater than that of the forward speed (Figures 8a and 10a). These uncertainties are expected due to variations in the location of the immersed obstacles. In each simulation, we allow the immersed obstacles to have uniformly distributed random positions. Figures $8 \mathrm{~b}$ and $10 \mathrm{~b}$ suggest that immersed obstacles will cause a turning maneuver to be coupled with the forward propulsion. The direction of turning will depend on the distribution of the immersed obstacles and can be controlled by exerting a controlled magnetic torque on the dipole moment of the spermbot.

\section{Discussion}

In this study, we develop a hydrodynamic model of the spermbot to predict its response in a viscous colloidal suspension. The model shows that the variability in the length of the mid-piece (variation in the length of the mid-piece is observed for different species ${ }^{[26]}$ and depth of the head inside the microtube influences the effective net motion of the spermbot. In particular, the forward swimming speed of the spermbot decreases with the length of the mid-piece of the sperm cell and the depth of the sperm head inside the microtube. Our theoretical model also predicts a fundamental difference between flagellar propulsion of the spermbot in a viscous media with and without immersed obstacles. The size and concentration of the immersed obstacles in the suspension have opposite effects on the forward swimming speed. Propulsion of the spermbot is enhanced by increasing the concentration of the immersed obstacles, while increasing the size of the obstacles decreases the forward speed. The propulsion enhancement is attributed to the local pressure field created at the distal tip of the beating flagellum. This field depends on the concentration of the obstacles and the velocity of the flow-field at the position of the obstacles. Spermbots and sperm cells create a relatively high flow-field in close proximity to the flagellum as opposed the microtube or head. Our simulation results reveal low fluid field velocities in front of the spermbot, while the flow velocity is relatively high behind the spermbot. This front-back asymmetry is even more influenced by the presence of immersed obstacles. Therefore, these obstacles create an asymmetric pressure gradient that contributes to the net propulsive force of the spermbot. The propulsion enhancement of the spermbot in a viscous medium with colloidal suspension can also be attributed 

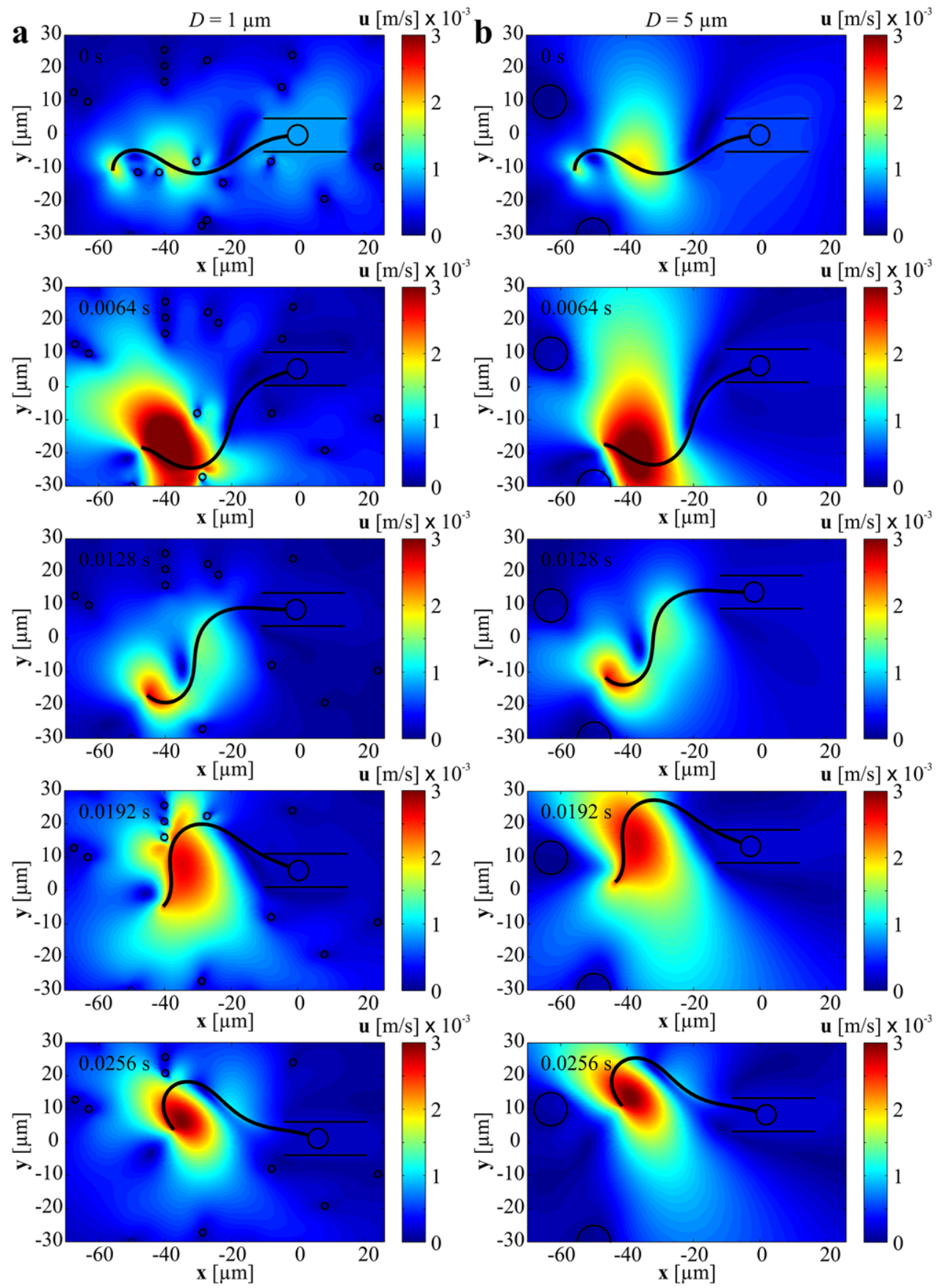

Figure 9. Influence of the size of the particles in the colloidal suspension on the flagellar propulsion of the spermbot is calculated for particles with diameter of 1 and $5 \mu \mathrm{m}$. The average speed of the spermbot decreases with the size of the particles in the colloidal suspension. Parameters: $\varphi=1 \%$, $L_{\mathrm{m}}=0 \mu \mathrm{m}$, and $d=10 \mu \mathrm{m}$. a) At $D=1 \mu \mathrm{m}, U_{x}=260 \pm 57$ and $U_{y}=48 \pm 28 \mu \mathrm{m} \mathrm{s}^{-1}$. b) At $D=5 \mu \mathrm{m}, U_{x}=128 \pm 50$, and $U_{y}=7 \pm 39 \mu \mathrm{m} \mathrm{s}$.

to the dependence of the drag coefficients on the concentration of the immersed obstacles. Gray and Hancock have shown that positive thrust force can only be achieved if the normal drag coefficient is greater than the tangential drag coefficient. ${ }^{[27]}$ This ratio is influenced by the size and concentration of the obstacles. The drag coefficient ratio $C_{\mathrm{n}} / C_{1}$ in the range $0<D \leq 2 \mu \mathrm{m}$, decreases nearly as $D^{-3}$. Above this range, the drag coefficient ratio is slightly affected by the size of the obstacles for a fixed concentration.
Overall, the forward velocities of the sperm cells and spermbots obtained by this model are higher than what is experimentally observed. We attribute this difference to factors that reduce the speed of the cells and spermbots such as interaction with surfaces, local friction in the vicinity of the microtube due to hydrophobicity of the involved materials. Additionally, it is commonly observed that sperm cells rotate inside the microtube. This also contributes to the decreased observed velocity of the spermbots. Furthermore, our model predicts the speed of the 

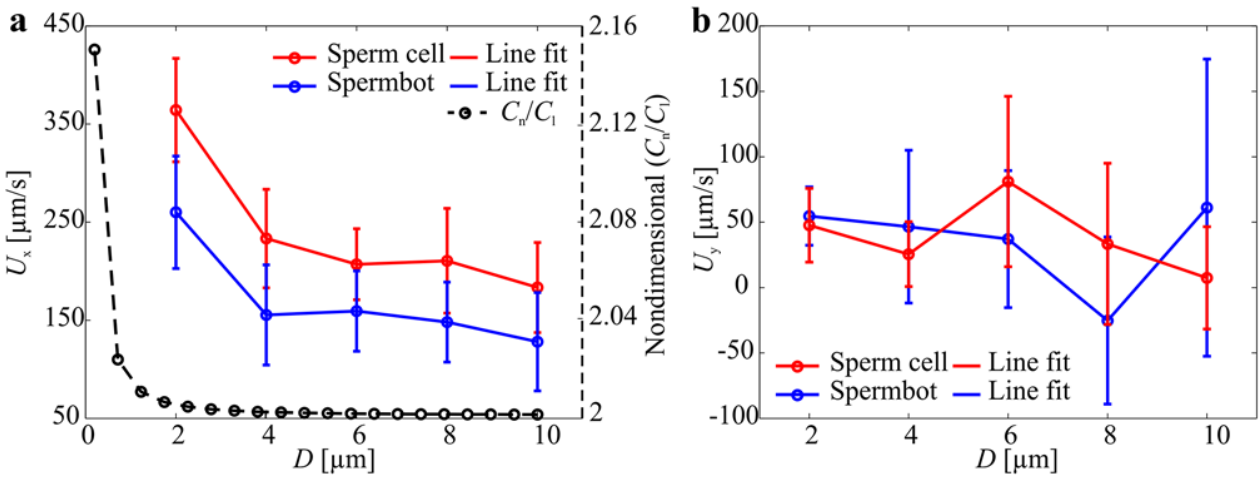

Figure 10. Forward and lateral speeds $\left(U_{x}\right.$ and $\left.U_{\gamma}\right)$ of the sperm and spermbots are calculated versus the diameter $(D)$ of the particles in the colloidal suspension. The particles are arranged randomly and the average speed and standard deviation are calculated for ten simulations. Parameters: $\varphi=1 \%$, $L_{\mathrm{m}}=0 \mu \mathrm{m}$, and $d=10 \mu \mathrm{m}$. a) The average forward speeds of the sperm and spermbot decrease by $12 \%$ and $12 \%$, respectively, for a $2 \mu \mathrm{m}$ increase in the diameter of the particles, respectively. b) The average lateral speed of the sperm and spermbot are not affected by the size of the immersed obstacles.

sperm cell and spermbot in 2D space, whereas sperm cells and spermbots swim in 3D space. Nevertheless, the model provides qualitative agreement with their real behavior in a viscous heterogeneous medium. A desirable modification to the current design of the spermbot would be to decrease the length of its microtube. The hydrodynamic model presented in this study suggests that the speed of the sperm cell is decreased by the microtube for two key reasons. First, the maximum forward speed of the spermbot is observed for relatively small depth of the head inside the microtube. Second, the microtube adds additional drag to the head and the mid-piece for the same propulsive force of the beating flagellum. The modification of the size of the microtube may decrease its average magnetic moment $(\mathbf{M})$, and influence the magnetic torque $(\mathbf{M} \times \mathbf{B})$ that counterbalances the drag torque during directional control. Therefore, it may be required to decrease the length of the microtube to enhance the propulsion of the spermbot, and increase the magnitude of the external magnetic field to compensate for the decreased magnetic moment. Likewise, the microtube length would affect the shape of flagella beating as the cargo load increases. For example, the work by Klindt et al., shows the influence of flow load on the beating of flagellated Chlamydomonas cells. ${ }^{[28]}$ Therefore, future modeling of the rate of hydrodynamic dissipation would be desirable to get more insights about the effect of increased load on the beat pattern when moving in colloidal suspensions. Wu et al. have also incorporated a liquid layer coating to minimize adhesion between micropropellers and the surrounding biopolymeric network, thereby improving propulsion. ${ }^{[29]}$ Therefore, it may be also required to incorporate a liquid layer to the magnetic constituent of the spermbot to enhance its propulsion.

\section{Acknowledgements}

This work was supported by the European Research Council under the European Union's Horizon 2020 Research and Innovation programme (Grant No. 638428-Project ROBOTAR: Robot-Assisted Flexible Needle Steering for Targeted Delivery of Magnetic Agents).

\section{Conflict of Interest}

The authors declare no conflict of interest.

\section{Keywords}

colloidal suspension, heterogenous viscous medium, microrobots, obstacles, propulsion, spermbots, stokeslets

Received: April 8, 2019

Revised: May 8, 2019

Published online: June 3, 2019

[1] V. Magdanz, S. Sánchez, O. G. Schmidt, Adv. Mater. 2013, 25, 6581.

[2] M. M. Stanton, J. Simmchen, X. Ma, A. Miguel-Lopez, S. Sanchez, Adv. Mater. Interfaces 2016, 3, 1500505.

[3] M. M. Stanton, B.-W. Park, A. Miguel-Lopez, X. Ma, M. Sitti, S. Sánchez, Small 2017, 13, 1603679.

[4] V. Magdanz, M. Medina-Sánchez, L. Schwarz, H. Xu, J. Elgeti, O. G. Schmidt, Adv. Mater. 2017, 29, 1606301.

[5] H. Xu, M. Medina-Sánchez, V. Magdanz, L. Schwarz, F. Hebenstreit, O. G. Schmidt, ACS Nano 2018, 12, 327.

[6] I. S. M. Khalil, V. Magdanz, S. O. Sanchez, O. G. Schmidt, S. Misra, J. Micro-Bio Robot. 2014, 9, 79.

[7] Y. Mei, G. Huang, A. A. Solovev, E. B. Ureña, I. Mönch, F. Ding, T. Reindl, R. K. Y. Fu, P. K. Chu, O. G. Schmidt, Adv. Mater. 2008, 20, 4085.

[8] V. Magdanz, M. Medina-Sánchez, Y. Chen, M. Guix, O. G. Schmidt, Adv. Funct. Mater. 2015, 25, 2763.

[9] M. Medina-Sánchez, M. Guix, L. Schwarz, O. G. Schmidt, in Proc. of the Int. Conf. on Manipulation, Automation and Robotics at Small Scales, Paris, France, July, 2016.

[10] V. Magdanz, M. Guix, F. Hebenstreit, O. G. Schmidt, Adv. Mater. 2016, 28, 4084.

[11] M. Medina-Sánchez, L. Schwarz, A. K. Meyer, F. Hebenstreit, O. G. Schmidt, Nano Lett. 2016, 16, 555.

[12] H. Xu, M. Medina-Sanchez, D. R. Brison, R. J. Edmondson, S. S. Taylor, L. Nelson, K. Zeng, S. Bagley, C. Ribeiro, L. P. Restrepo, E. Lucena, C. K. Schmidt, O. G. Schmidt, arXiv:1904.12684 [q-bio.CB], 2019.

[13] M. Medina-Sánchez, O. G. Schmidt, Nature 2017, 545, 406.

[14] M. Medina-Sánchez, V. Magdanz, L. Schwarz, H. Xu, O. G. Schmidt, Conf. on Biomimetic and Biohybrid Systems, Stanford, CA, July 2017.

[15] R. H. F. Hunter, P. Coy, J. Gadea, D. Rath, J. Assist. Reprod. Genet. 2011, 28, 191.

[16] J. C. Kirkman-Brown, D. J. Smith, Mol. Hum. Reprod. 2011, 17, 539.

[17] H. C. Berg, L. Turner, Nature 1979, 278, 349.

[18] A. M. Leshansky, Phys. Rev. E 2009, 80, 051911.

[19] J. L. Münch, D. Alizadehrad, S. B. Babu, H. Stark, Soft Matter 2016, 12,7350 
[20] A. Zöttl, J. Yeomans, Nat. Phys. 2019, 11 https://doi.org/10.1038/ s41567-019-0454-3.

[21] R. Cortez, SISC 2001, 23, 1204.

[22] R. Cortez, L. Fauci, A. Medovikov, Phys. Fluids 2005, 17, 031504.

[23] B. Rodenborn, C.-H. Chen, H. L. Swinney, B. Liu, H. P. Zhang, Proc. Natl. Acad. Sci. U. S. A. 2013, 110, E338.

[24] J. L. Perkins, L. Goode, J. Animal Sci. 1966, 25, 465.
[25] B. M. Friedrich, I. H. Riedel-Kruse, J. Howard, F. Jülicher, J. Exp. Biol. 2010, 213, 1226.

[26] R. A. Cardullo, J. M. Baltz, Cell Motil. Cytoskeleton 1991, 19, 180.

[27] J. Gray, H. J. Hancock, J. Exp. Biol. 1955, 32, 802.

[28] G. Klindt, C. Ruloff, C. Wagner, B. M. Friedrich, Phys. Rev. Lett. 2016 $117,258101$.

[29] Z. Wu, J. Troll, H.-H. Jeong, Q. Wei, M. Stang, F. Ziemssen, Z. Wang, M. Dong, S. Schnichels, T. Qiu 1, P. Fischer, Sci. Adv. 2018, 4, eaat4388. 